\title{
The Luminosity Function of Galaxies in Compact Groups
}

\author{
S. D. Hunsberger
}

Lowell Observatory, Flagstaff, AZ, USA

\section{J. C. Charlton}

Pennsylvania State University, University Park, PA, USA

D. Zaritsky

University of California/Lick Observatory, Santa Cruz, CA, USA

\begin{abstract}
From R-band images of 39 Hickson compact groups (HCGs), we use galaxy counts to determine a luminosity function. We find that a single Schechter function is a poor fit to the data, so we use a composite function consisting of separate Schechter functions for the bright and faint galaxies. The decreasing bright end slope implies a deficit of intermediate luminosity galaxies in our sample and the faint end is slightly steeper than that reported for earlier HCG luminosity functions. Furthermore, luminosity functions of subsets of our sample reveal more substantial dwarf populations for groups with X-ray halos, groups with tidal dwarf candidates, and groups with a dominant elliptical or lenticular galaxy. Collectively, these results support the hypothesis that within compact groups, the initial dwarf galaxy population is replenished by "subsequent generations" formed in the tidal debris of giant galaxy interactions.
\end{abstract}

\section{Introduction}

The earliest compact group luminosity functions (Heiligman \& Turner 1980, Mendes de Oliveira \& Hickson 1991) predicted a lack of dwarf galaxies and it was assumed that due to the high number density of galaxies and frequency of interactions, the compact group environment was too hostile for dwarf galaxies to survive. More recently, galaxy counts from deeper imaging of HCGs (Ribeiro et al. 1994, Zepf et al. 1997, Hunsberger et al. 1998) indicate that there are dwarf galaxy members in compact groups.

\section{Observations and Data Analysis}

We obtained R-band images of 39 HCGs at the Palomar 1.5m telescope using a Tektronix CCD which provided a $12.8^{\prime} \times 12.8^{\prime}$ field of view and a resolution of $0.75^{\prime \prime}$ per pixel. We restricted our HCG sample to groups with angular diameters 

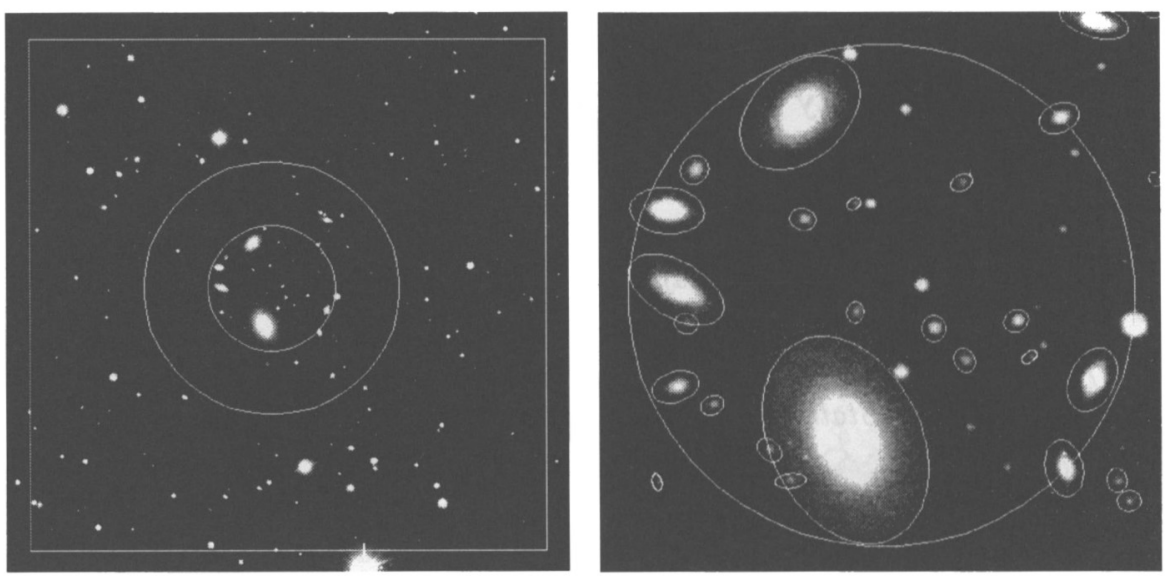

Figure 1. On the left is an image of HCG \#12. The smaller circle marks the area of the compact group. The larger circle marks the inner boundary of the background region. On the right is a close-up view of the same group highlighting the FOCAS detections of faint galaxies. Some of these objects are group members while others are background galaxies. Statistically, we can quantify the number of HCG members, but cannot determine membership for individual objects.

$<7^{\prime}$ so that they fit well within the CCD frame and with $z \leq 0.05$ so that faint dwarf galaxies could be detected during a total exposure time of 30 minutes.

We determine a luminosity function with a statistical technique that involves using galaxy counts in the outer regions of CCD images to estimate the background/foreground contribution to galaxy counts in the compact group region. For each magnitude interval, the compact group members are measured as an excess above the expected background galaxy counts. We used FOCAS (Faint Object Classification and Analysis System) (Jarvis \& Tyson 1981) to identify galaxies, calculate magnitudes, and determine positions. Figure 1 illustrates the definition of "background" and "group" regions and highlights FOCAS identification of faint galaxies on an HCG image.

\section{Results}

Our luminosity function extends approximately two magnitudes fainter than previous luminosity functions. We fit the luminosity function with a composite of separate Schechter functions for the bright and faint galaxies (see Figure 2a). The decreasing bright end slope hints at a deficit of intermediate luminosity galaxies in our sample of HCGs and the faint end slope is slightly higher than that reported for earlier HCG luminosity functions.

By calculating luminosity functions for various sub-samples of HCGs, we discover that groups with candidate tidal dwarf galaxies (Hunsberger et al. 1996), groups with X-ray halos (Ponman et al. 1996), and groups with a dominant elliptical or S0 galaxy (Hickson 1982) have significantly larger dwarf pop- 
(a) LF of HCG Goloxies

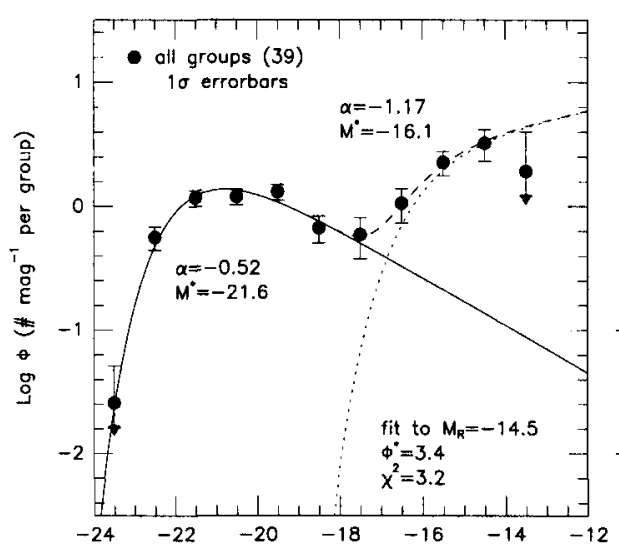

(c) LFs of groups with and without

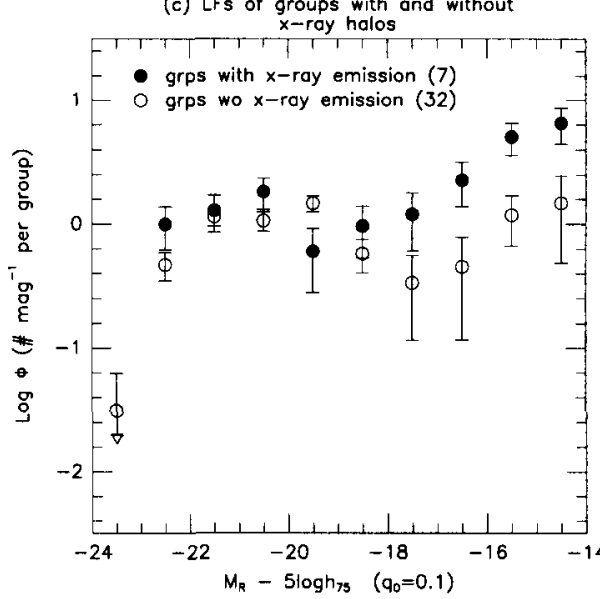

(b) LFs of groups with and without

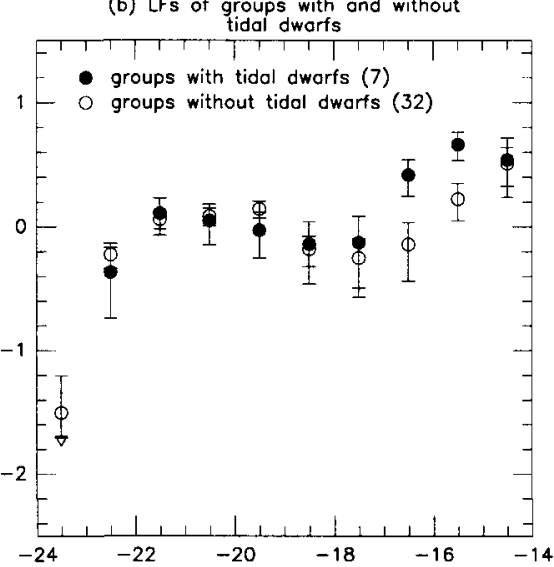

(d) LFs of groups with and without a dominant $E / S O$ galaxy

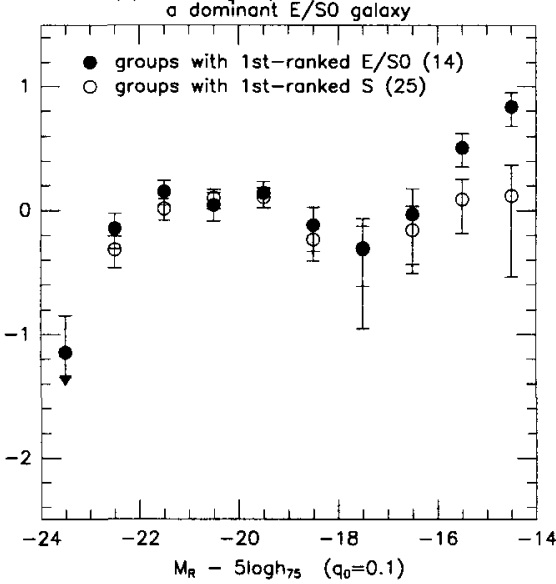

Figure 2. In all plots, the data points represent the average number of galaxies per magnitude interval per compact group. The numbers in parentheses near the top of each plot indicate the number of groups in each subset. Because the ultimate fate of tidal dwarfs is unknown, they are not included in the luminosity functions for (c) and (d). For the luminosity function of the entire sample (a), the bright and faint populations are fit separately using 2 Schechter functions. The solid line is the bright end, the dotted line is the faint end, and the dashed line is the composite fit. For luminosity functions of various subsets (b, $c, d)$, there is an excess of dwarf galaxies in groups with tidal dwarfs, groups with X-ray halos, and groups with a dominant elliptical when compared to compact groups without these properties. 
ulations than groups without these properties. Because such characteristics are associated with interactions and mergers, this discovery supports the hypothesis that the initial dwarf galaxy population is augmented by "subsequent generations" formed in the tidal debris of giant galaxy interactions. A comparison of luminosity functions for groups with and without these properties is shown in Figures 2b-d. We also present the relevant information in tabular form below.

Table 1. Compact Group Populations

\begin{tabular}{|lcrrr|}
\hline \multicolumn{1}{|c}{$\begin{array}{c}\text { Giants } \\
\text { Description }\end{array}$} & $\begin{array}{c}\text { Dwarfs } \\
\text { per } \\
\text { Group }\end{array}$ & \multicolumn{1}{c|}{$\begin{array}{c}\text { Der } \\
\text { Group }\end{array}$} & $\begin{array}{c}\text { perfs } \\
\text { Giant }\end{array}$ & $P_{\chi}{ }^{b}$ \\
\hline all groups (39) & 4.2 & $7.2 \pm 1.1$ & $1.7 \pm 0.3$ & \\
\hline with tidal dwarfs (7) & 4.1 & $11.4 \pm 2.3$ & $2.8 \pm 0.6$ & 0.013 \\
without tidal dwarfs (32) & 4.2 & $6.2 \pm 1.3$ & $1.5 \pm 0.3$ & \\
\hline with 1st-ranked E/S0 $^{c}(14)$ & 4.7 & $11.5 \pm 2.4$ & $2.4 \pm 0.5$ & 0.012 \\
with 1st-ranked Sc $(25)$ & 3.9 & $3.7 \pm 1.3$ & $0.9 \pm 0.3$ & \\
\hline with X-ray emission $^{c}(7)$ & 4.6 & $15.0 \pm 2.8$ & $3.3 \pm 0.6$ & $<0.001$ \\
without X-ray emission $^{c}(32)$ & 4.1 & $3.4 \pm 1.2$ & $0.8 \pm 0.3$ & \\
\hline
\end{tabular}

${ }^{\alpha}$ Dwarf galaxy counts include objects in the luminosity range $-18.0<M_{R}-5 \log h_{75}<-14.0$. ${ }^{b}$ Calculated values are based on a comparison of faint end data points of the luminosity functions using a generalized $\chi^{2}$ test. A value of $P_{\chi}$ approaching zero implies different populations.

${ }^{c}$ Tidal dwarf candidates are not included in these subsets.

From these results, we speculate that the evolution of a typical HCG may proceed as follows: The group forms because dynamical friction of massive dark halos around field galaxies brings them together. These newly formed HCGs contain dwarf companions to the giant galaxies, but these are gradually cannibalized as interactions occur. Eventually a major merger occurs and this has several consequences: the formation of dwarf galaxies in tidal debris, the production of an X-ray halo, and the formation of a giant $\mathrm{E} / \mathrm{S} 0$ galaxy. The ongoing interactions strip gas, dark matter, and dwarf galaxies from the giants and spread them into a common group halo. ing.

Acknowledgments. The IAU kindly provided travel assistance to the meet-

\section{References}

Heiligman, G. M. \& Turner, E. L. 1980, ApJ, 236, 745

Hickson, P. 1982, ApJ, 255, 382

Hunsberger, S. D., Charlton, J. C., \& Zaritsky, D. 1996, ApJ, 462, 50

Hunsberger, S. D., Charlton, J. C., \& Zaritsky, D. 1998, ApJ, 505, 536

Jarvis, J. F. \& Tyson, J. A. 1981, AJ, 86, 476

Mendes de Oliveira, C. \& Hickson, P. 1991, ApJ, 380, 30

Ponman, T. J., Bourner, P. D. J., Ebeling, H., \& Bohringer, H. 1996, MNRAS, 283, 690 
Ribeiro, A. L. B., de Carvalho, R. R., \& Zepf, S. E. 1994, MNRAS, 267, L13 Zepf, S. E., de Carvalho, R. R., \& Ribeiro, A. L. 1997, ApJL, 488, 11 